\title{
A prospective single centre study comparing computed tomography pneumocolon against colonoscopy in the detection of colorectal neoplasms
}

\author{
Y M Miao, Z Amin, J Healy, P Burn, N Murugan, D Westaby, T G Allen-Mersh
}

\begin{abstract}
Background and aims-To determine the sensitivity and specificity of computed tomography (CT) pneumocolon in the detection of colorectal neoplasms.

Methods-A total of 201 consecutive patients with colorectal symptoms or requiring surveillance for colorectal neoplasms underwent both conventional colonoscopy and CT pneumocolon.

Results-On conventional colonoscopy 13 invasive colorectal carcinomas were detected in 13 patients, and 118 polyps in 63 patients (14 polyps were $\geqslant 1 \mathrm{~cm}$ in diameter, 25 were $6-9 \mathrm{~mm}$, and 79 were $\leqslant 5$ $\mathrm{mm})$. CT pneumocolon detected all 13 cancers, two false positive cancers, but only 20 polyps (seven were $\geqslant 1 \mathrm{~cm}$ ). This resulted in a sensitivity of $100 \%(95 \%$ confidence interval (CI) $87-100 \%$ ) and specificity of $99 \%(95 \%$ CI $97-100 \%)$ for detection of invasive carcinoma, and a sensitivity of $73 \%(95 \%$ CI $56-90 \%)$ and specificity of $94 \%$ (95\% CI $91-98 \%)$ for detection of invasive carcinoma and/or $\geqslant 1$ cm polyps. CT pneumocolon also identified invasive carcinoma not seen at colonoscopy because of incomplete examination in three patients, and detected metastases in six colorectal carcinoma patients and extracolonic carcinoma in a further seven patients.
\end{abstract}

Conclusions-CT pneumocolon had a high sensitivity and specificity for detection of invasive colorectal carcinoma but not colorectal polyps. CT pneumocolon may be suitable for initial investigation of patients with symptoms of colorectal malignancy.

(Gut 2000;47:832-837)

Keywords: computed tomography; colonoscopy; colorectal cancer; colonic polyps

Conventional colonoscopy is currently the most accurate means of identifying colorectal neoplasms ${ }^{1}$ and has the advantage of allowing biopsy and polypectomy. However, conventional colonoscopy may visualise the entire colon in only $60-90 \%$ of patients ${ }^{23}$ and may produce colonic perforation in $0.03-0.65 \%$ of non-therapeutic procedures. ${ }^{4}$ Therefore, there is a potential role for a non-invasive technique which can image the entire colon and reliably identify colonic neoplasms. One promising approach involves computed tomography (CT) of the colon.

Various names have been given to different CT colon techniques, including CT pneumocolon, ${ }^{5} \mathrm{CT}$ colography, ${ }^{6}$ and virtual colonoscopy. ${ }^{8}$ In all cases the colon is first emptied and spiral CT scans of the abdomen and pelvis are performed following rectal insufflation. Virtual colonoscopy is essentially a low milliamps $(\mathrm{mA})$ technique that relies on thin collimation, no intravenous contrast, and three dimensional endoluminal computerised reconstruction and visualisation, whereas CT pneumocolon uses thicker collimation, intravenous contrast but omits three dimensional imaging. Virtual colonoscopy gives high quality images of the colonic lumen but suboptimal assessments of extracolonic pathology which can be a drawback of this technique. Additionally, the large numbers of two dimensional axial images increases the time needed for data reconstruction and viewing, and extra software is required for three dimensional reconstruction. Spiral CT pneumocolon, which is a simple extension of conventional abdominal and pelvic CT, does not produce excessive images and no specialised software is required.

The aim of the study was to determine the accuracy of CT pneumocolon in detecting colorectal neoplasms identified at conventional colonoscopy.

\section{Materials and methods}

This was a prospective single centre study. Ethics approval was obtained from the Riverside Research Ethics Committee, Chelsea and Westminster Hospital, London. Patients attending one hospital between February 1998 and September 1999 requiring colonoscopy for colorectal symptoms or surveillance of colorectal neoplasia were asked to participate. Exclusion criteria were a history of inflammatory bowel disease, family history of colorectal cancer in an asymptomatic patient, and age less than 55 years. The lower age limit of 55 years was stipulated to reduce any long term radiation risk from CT. All patients received standard bowel preparation involving either up to 4 litres of a polyethylene glycol-electrolyte solution or two sachets of sodium picosulphate with magnesium citrate mixed in water.

Abbreviations used in this paper: $\mathrm{CT}$, computed tomography. 
CT PNEUMOCOLON

Patients were placed in the left lateral position and a rectal tube inserted. Intravenous hyoscine butylbromide $(20 \mathrm{mg})$ was administered and the colon gently insufflated with carbon dioxide until the patient experienced mild abdominal discomfort or to a maximum volume of 2 litres; this was undertaken by trainee radiologists. A supine CT scanogram (Siemen Somatom Plus 4, Erlangen, Germany) was performed. Colonic distension was assessed by the radiologist from this image and further room air administered if collapsed segments were seen. Intravenous iohexol $(100 \mathrm{ml})$ (300 mgI/ml; Nycomed Amersham plc, Little Chalfont, Buckinghamshire, UK) was injected at a rate of $3 \mathrm{ml} / \mathrm{s}$ using a Liebel/Flarsheim pressure CT 9000 injector (Cincinnati, Massachusetts, USA). Scanning was started 25 seconds later passing caudocranially from symphysis pubis to diaphragm. A collimation of $8 \mathrm{~mm}$ with a pitch of 1.5 and a reconstruction interval of $4 \mathrm{~mm}$ were used to limit the radiation dose to a level comparable with that of a barium enema (5-7 $\mathrm{mSv}$ ) and to minimise the number of images to allow rapid interpretation. The CT pneumocolon was performed within a single breath hold - the breath was held once the scan level passed above the true pelvis. If rectal and sigmoid distension was deemed inadequate after initial scanning, the patient was turned to the prone position and scans of the pelvis repeated without further intravenous contrast. In practice, both supine and prone pelvis views were obtained in the majority of patients.

Interpretation of the scan images using a computerised workstation (Sienet MagicView 1000, Siemens, Erlangen, Germany) was performed independently by two trained radiologists - each had more than five years' experience in reporting abdominal CT scans. Images were viewed on soft tissue, lung, and intermediate windows. Where there was disagreement in reporting, a consensus was reached by discussion between the two radiologists. A note was made of the time taken for scanning and reporting each colon CT.

Throughout the entire study any feedback of the colonoscopy results was withheld from the reporting radiologists to avoid a learning bias that might improve the accuracy of CT pneumocolon during the course of the study. After the last study had been reported, a retrospective review of CT images was undertaken to establish reasons for disagreement with colonoscopy.

CONVENTIONAL COLONOSCOPY

Patients underwent conventional colonoscopy at least one hour after completion of the CT scan. Patients were sedated with a combination of intravenous midazolam and pethidine. Colonoscopies were performed by one of 12 gastroenterologists, each with more than three years colonoscopy experience, using standard video colonoscopes (Olympus Keymed CF 200-HL, Southend-on-Sea, UK). The endoscopist was not told the CT pneumocolon results. The estimated level of colon reached at
$60 \mathrm{~cm}$ from the anal verge (the extent reached by flexible sigmoidoscopy), and the final level reached were noted. Abnormalities were recorded at each level. Polyp size was estimated against biopsy forceps or by direct measurement after retrieval following polypectomy. Duration and patient tolerance of the procedure were noted.

Patients were asked to score the pain experienced from each procedure using a visual analogue score of $1-5$ ( 1 , no pain; 5 , very painful). The score for CT pneumocolon was completed immediately after the procedure while that for conventional colonoscopy was recorded after recovery from sedation. Within a month of examination, all patients were sent follow up questionnaires enquiring about their preference for the two procedures.

For data analysis the colon was divided into four segments: right, transverse, left, and rectosigmoid. Agreement was evaluated between the two techniques based on the size and location of the neoplasm. If colonoscopy was incomplete, results were compared with the limit of the examination. Neoplasms identified by CT pneumocolon that were situated in segments not visualised by the study conventional colonoscopy were excluded from the comparative analysis.

A review of each patient's clinical notes was undertaken after the study (median interval from study 11 months, interquartile range (IQR) 6-15) to establish if colorectal neoplasia was identified by subsequent investigations (including repeat colonoscopy) in patients whose initial study colonoscopy did not demonstrate a colorectal neoplasm.

\section{STATISTICAL CONSIDERATIONS}

The primary objective of the study was to establish the extent to which CT pneumocolon might fail to identify colonic neoplasms. Therefore, we determined that the study should have a mean sensitivity of $98.5 \%$ with a lower $95 \%$ confidence interval (95\% CI) of $95 \%$ for CT pneumocolon in identification of colorectal neoplasms against the conventional colonoscopy "gold standard". Assuming that a colorectal neoplasm is identified on colonoscopy in $25 \%$ of the patient group, 200 patients would be required for the lower 95\% confidence limit of a mean sensitivity of $98.5 \%$ not to fall below 95\% (normal approximation to the binomial distribution). Sensitivities and specificities were calculated on a cumulative basis, starting with invasive carcinoma and then including polyps by diminishing diameter. Confidence intervals for sensitivity and specificity were calculated using the normal approximation to the binomial distribution. However, where values of $0 \%$ or $100 \%$ were obtained, the exact binomial distribution was used, with a probability parameter (p) of 0.01 or 0.99 , respectively. The significance of differences in polyp detection rates by size and location were assessed by contingency tables using Pearson's $\chi^{2}$ statistic and Cochran's test for a linear trend. Pain score and procedure duration differences were assessed by Mann-Whitney U test. 
Table 1 Identification by CT pneumocolon of colorectal polyps diagnosed by conventional colonoscopy in the 201 patients studied. Results are shown on both a "per patient" and "per polyp" basis by polyp diameter

\begin{tabular}{|c|c|c|c|c|}
\hline Polyp diameter & $n$ & True positive & False negative & False positive \\
\hline Analysis per patient ${ }^{\star}$ & $(p t s)$ & $(p t s)$ & (pts) & (pts) \\
\hline$\geqslant 10 \mathrm{~mm}$ polyp & $13(1)$ & $6(1)$ & 7 & 8 \\
\hline 6-9 mm polyp & 15 & 3 & 12 & 4 \\
\hline$\leqslant 5 \mathrm{~mm}$ polyp & $31(3)$ & 5 & $26(3)$ & 4 \\
\hline All polyps & $59(4)$ & $14(1)$ & $45(3)$ & 16 \\
\hline Analysis per polyp & (polyps) & (polyps) & (polyps) & (polyps) \\
\hline$\geqslant 10 \mathrm{~mm}$ polyp & 14 & 7 & 7 & 8 \\
\hline 6-9 mm polyp & 25 & 4 & 21 & 6 \\
\hline $1-5 \mathrm{~mm}$ polyp & 79 & 9 & 70 & 11 \\
\hline All polyps & 118 & 20 & 98 & 25 \\
\hline
\end{tabular}

* These values represent the largest polyp detected in patients without invasive colorectal cancer. Patients with both invasive colorectal carcinoma and colorectal polyps are given in parentheses. $\mathrm{n}$, number, pts, patients.
$60 \mathrm{~cm}$ of the anal margin. Ninety four polyps were retrieved for histological examination and the majority of the 24 polyps that were not examined histologically were $\leqslant 5 \mathrm{~mm}$ in diameter. Histology revealed adenocarcinoma in situ in a single $1 \mathrm{~cm}$ polyp within the descending colon.

\section{Other abnormal findings}

Conventional colonoscopy also detected caecal angiodysplasia (three patients), Crohn's disease (one), and ulcerative colitis (one).

\section{Complications}

Two patients required reversal of sedation with flumazenil and three required intravenous atropine following bradycardia and hypotension during the colonoscopy procedure. No other morbidity or mortality was associated with colonoscopy.

A total of 233 patients were recruited. Thirty two patients did not complete the study; of these, 16 patients withdrew after agreeing to participate, 11 did not attend their appointments, and one was cancelled by the responsible medical team. There were four protocol violations: one patient was found to be aged less than 55 years due to an error in birth date registration, and three underwent CT pneumocolon but did not have subsequent colonoscopy (two refused and one was too ill for sedation). Results from the remaining 201 patients (83 males, 118 females; mean age 71 years (range 55-91)) are reported. Indications for conventional colonoscopy in these patients were: change in bowel habit, 91; abdominal pain, 65; rectal bleeding, 53; polyp surveillance, 32; invasive carcinoma surveillance, 25; suspected abdominal mass, 23; anaemia, 23; weight loss, 15; abnormal barium enema or abdominal ultrasound, eight.

\section{CONVENTIONAL COLONOSCOPY}

A complete colonoscopic examination to the caecum or beyond was possible in $170(85 \%)$ patients. Reasons for failure in the remaining 31 patients were: poor bowel preparation (12 patients), unsuitable bowel configuration (11), obstructing neoplasm (six), and poor patient tolerance (two). Colonoscopy was repeated in six of the 12 patients in whom bowel preparation was inadequate. Two of these patients were found to have invasive colorectal carcinoma at repeat colonoscopy, both situated proximal to the level reached on initial colonoscopy. These two invasive carcinomas are not included in comparisons with CT pneumocolon as they were not identified at the time of the study colonoscopy.

Complete colonoscopy was normal in 111 patients, and a further 18 patients had normal limited colonoscopy.

\section{Colorectal neoplasms}

Seventy two patients (36\%) were found to have colorectal neoplasms on conventional colonoscopy. Thirteen of these patients had 13 invasive colorectal carcinomas while 118 polyps were found in 63 patients (four also had invasive carcinoma) (table 1). All invasive carcinomas and most $(82 / 118)$ polyps were situated within or distal to the left side of the colon and within
CT PNEUMOCOLON

All 201 patients underwent the CT pneumocolon examination. Colon segments were collapsed in 32 patients ( 24 patients had one collapsed segment; five patients had two; one patient had three; and two patients had four). This collapse affected the rectosigmoid (27 patients), left (14), transverse (two), and right (five) colon segments.

A total of 126 patients had a technically satisfactory examination that was reported as normal.

\section{Detection of colorectal neoplasms}

Colonic neoplasms were detected by CT pneumocolon in 27 (13 cancers and 14 polyps) of the 72 patients in whom neoplasms were identified by the study colonoscopy. CT pneumocolon detected one patient with both invasive colorectal carcinoma and $a \geqslant 1 \mathrm{~cm}$ polyp but missed small $\leqslant 5 \mathrm{~mm}$ polyps in three patients who also had invasive colorectal carcinoma. This resulted in a sensitivity of $38 \%$ (95\% CI $26-49 \%)$ and specificity of $86 \%(95 \%$ CI $80-92 \%$ ) for detection by CT pneumocolon of the most clinically relevant colonic neoplasm per patient (table 1).

\section{Detection of invasive colorectal carcinoma}

CT pneumocolon identified colonic tumours 18 patients. This included the 13 patients found to have invasive colonic carcinoma and two patients who did not have invasive carcinomas. An additional three colonic tumours were detected in the colon (right, transverse, and rectosigmoid segments) of patients who had undergone limited colonoscopy because of poor bowel preparation. Two of these patients subsequently underwent repeat colonoscopy after further bowel preparation, and the colonic tumours seen on CT pneumocolon (fig 1) were confirmed on biopsy as adenocarcinomas. The diagnosis of adenocarcinoma in the third patient, whose CT pneumocolon suggested a sigmoid tumour adherent to the bladder, was confirmed after surgical resection. Since the accuracy of CT pneumocolon was based on a comparison of CT against colonoscopy in the that were suspicious of invasive carcinoma in 


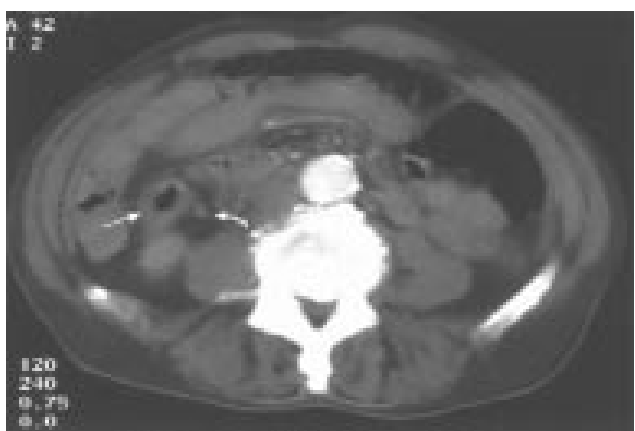

Figure 1 CT pneumocolon shows circumferential thickening of the colon just below the hepatic flexure (straight arrow) with some irregularity of the outer wall and a small adjacent lymph node (curved arrow). These features are consistent with a colonic cancer on CT. This tumour was not seen on initial colonoscopy because of poor bowel preparation but was confirmed on subsequent colonoscopy.

segments of colon adequately visualised by the study conventional colonoscopy, these additional three invasive cancers were not included in estimates of accuracy. On this basis, the sensitivity of CT pneumocolon for detection of invasive colorectal carcinoma was 100\% (95\% CI $87-100 \%)$ and specificity was $99 \%(95 \%$ CI $97-100 \%$ ).

\section{Detection of colorectal polyps}

Results on a "per patient" and a "per polyp" basis by polyp diameter are shown in table 1 . There was a significant increase (Cochran's test for linear trend in a $3 \times 2$ contingency table; $\mathrm{p}=0.0015)$ in the probability of CT pneumocolon polyp detection by increasing polyp diameter ( $1-5 \mathrm{~mm}, 9 / 79$ polyps; $6-9 \mathrm{~mm}, 4 / 25 ; \geqslant 10$ $\mathrm{mm}, 7 / 14$ ) but not by polyp location (Pearson's $\chi^{2}$ test in a $4 \times 2$ contingency table) (right colon, $1 / 26$ polyps; transverse, $2 / 10$; left, $4 / 17$; rectosigmoid, 13/65). This resulted in a progressively decreasing sensitivity and specificity when smaller polyps were included in calculations of accuracy (invasive carcinoma and/or $\geqslant 10 \mathrm{~mm}$ polyps, sensitivity $73 \%$ (95\% CI 56-90\%), specificity 94\% (95\% CI 91-98\%); invasive carcinoma, $\geqslant 10 \mathrm{~mm}$ and/or 6-9 $\mathrm{mm}$ polyps, sensitivity $54 \%$ (95\% CI 38-69\%), specificity $91 \%$ (95\% CI $87-96 \%)$; invasive carcinoma and/or all polyps, sensitivity 38\% (95\% CI 26-49\%), specificity $86 \%$ (95\% CI $80-92 \%))$

Detection of extracolonic abnormalities

Of the 16 patients with invasive colorectal cancer, nine showed evidence of local fat infiltration and six had distant metastases (five liver, one ascites). A further three patients undergoing surveillance for previous colorectal carcinoma resections had metastases at four sites (two liver, one kidney, one lung base) despite normal colonoscopies. Unsuspected pancreatic cancer (three patients), renal cancer (one), ileal Crohn's disease (one), and a dissecting thoracic aortic aneurysm (one) were also identified.

\section{Complications}

No morbidity or mortality was associated with the CT pneumocolon examination.
PROCEDURE TIMES FOR CONVENTIONAL COLONOSCOPY AND CT PNEUMOCOLON

The procedure time was significantly shorter (Mann-Whitney U, p <0.001) for conventional colonoscopy (median 25 minutes (IQR 1535)) compared with CT pneumocolon (33 minutes (IQR 25-45)). However, these results do not include the recovery time following sedation required for conventional colonoscopy. This varied depending on whether the patient was an inpatient or outpatient and on the time of patient collection from the endoscopy department. The median CT pneumocolon reporting time was 10 minutes (IQR 10-10).

PATIENT PAIN SCORES AND PREFERENCE

Patient recorded pain score was significantly less (Mann-Whitney U, p<0.001) for conventional colonoscopy (median 1 (IQR 1-2)) compared with CT pneumocolon (median 3 (IQR 2-3)). The follow up questionnaire indicated that 83 patients preferred CT pneumocolon; 94 preferred conventional colonoscopy; 20 had no preference; and one disliked both. Three patients did not reply.

RETROSPECTIVE REVIEW OF CT PNEUMOCOLON IMAGES AGAINST COLONOSCOPY FINDINGS The abnormalities seen on the scans from the two patients with false positive reports of invasive colorectal cancer by CT pneumocolon were attributed to inadequate distension of the caecum in the first patient and thickened haustral folds in the second. Low rectal polyps were partially obscured by the rectal insufflation tube in two patients, and these were probably visible on retrospective review of CT films (fig 2). The pedunculated polyp containing adenocarcinoma in situ was also probably visible with hindsight, at the junction of the sigmoid and descending colon. The remaining four of the seven missed $\geqslant 1 \mathrm{~cm}$ diameter polyps were not visible on retrospective review of CT films. In three of these cases collapse of the rectosigmoid segment was likely to have obscured the polyp, and in one case a caecal polyp was not visible due to the presence of faecal fluid. The clinical notes were reviewed in the eight patients with $\geqslant 1 \mathrm{~cm}$ polyps which were reported on CT pneumocolon but not trial

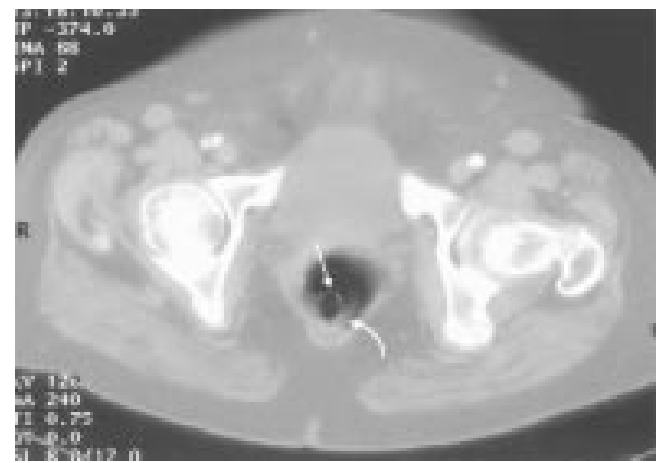

Figure 2 CT pneumocolon at the level of the rectal tube (straight arrow). This patient had a $1 \mathrm{~cm}$ rectal polyp seen at conventional colonoscopy but not reported on $C T$

pneumocolon. On retrospective review, the only abnormality detected is shown adjacent to the rectal tube (curved arrow) and is thought to possibly represent the polyp. 
colonoscopy. Three patients had repeat endoscopic examinations to determine if the polyps seen on CT had been missed. None of these patients was found to have the suggested $\geqslant 1$ $\mathrm{cm}$ polyp seen on the CT pneumocolon although $<5 \mathrm{~mm}$ hyperplastic polyps were found in the region of abnormality reported. In the remaining five patients, the responsible clinical team decided not to repeat colonoscopy. There was good colonic distension at CT pneumocolon in all patients with false positive reports of polyps. False positive results arose from misinterpretation of hypertrophied haustral folds associated with sigmoid diverticular disease, faecal residue, or a prominent ileocaecal valve.

\section{Discussion}

In this study, gastroenterologists with only three years' colonoscopy experience performed conventional colonoscopy and this may explain the $15 \%$ failure rate to reach the caecum. Similarly, radiology trainees undertook the rectal insufflation necessary for CT pneumocolon and good colonic distension was not always achieved. Although better results may have been achieved by more experienced personnel, the current results reflect the accuracy achieved in routine practice.

In our study, CT pneumocolon had a poor overall sensitivity for detecting colonic neoplasms (38\%, 95\% CI 26-49\%) which was well below the stipulated lower limit of $95 \%$. However, CT pneumocolon had a high sensitivity and specificity for detection of invasive colorectal carcinoma. In addition, information on local tumour extension and metastatic disease that was not available from conventional colonoscopy was provided by CT pneumocolon at the time of tumour identification. The CT technique did not require specialised software and was performed with acceptable procedure and reporting times. Despite this, these promising results should be interpreted with caution as invasive carcinomas were a subgroup of the neoplasm group and the incidence of invasive carcinomas was lower than that specified in the initial power calculations. Although we recorded $100 \%$ sensitivity for detection of invasive cancers, the lower 95\% confidence interval of this estimate was $87 \%$ which would be regarded as unacceptable. Assuming that $8 \%$ of patients had invasive colorectal carcinoma (as in this study), 625 patients would need to be studied for the lower $95 \%$ confidence limit of a $98.5 \%$ sensitivity rate not to fall below $95 \%$.

Detection of $\geqslant 1 \mathrm{~cm}$ diameter colorectal polyps is clinically more important than detection of smaller polyps which have a relatively low malignant potential (approximately 1\%), with progression to invasive malignancy usually requiring an interval of 10 years. ${ }^{9}$ We found that the probability of polyp detection by CT pneumocolon significantly increased with polyp diameter. An in vitro study of polyp detection in a pig colon by Dachman et al suggested that simulated $10 \mathrm{~mm}$ polyps were clearly visible with a $7 \mathrm{~mm}$ collimation and a pitch of up to 2 on initial (non-reconstructed) images. ${ }^{10}$ We hypothesised that detection of $\geqslant 1$ $\mathrm{cm}$ polyps would be feasible with a collimation of $8 \mathrm{~mm}$, pitch of 1.5 , and reconstruction interval of $4 \mathrm{~mm}$, provided the colon was adequately emptied and distended. These parameters allowed supine scanning to be completed in a single breath hold in virtually all patients, with a radiation dose which was similar to that of conventional barium enema (5-7 $\mathrm{mSv}$ ). In the present study, the $73 \%$ sensitivity for detection of $\geqslant 1 \mathrm{~cm}$ polyps and/or invasive carcinomas on a per patient basis was similar to that previously reported $(75-91 \%)$ in some studies $^{611}$ using thinner collimation and reconstruction intervals, and three dimensional reconstruction with specialised computer software. However, Rex et al recently evaluated two dimensional and three dimensional colography in 46 asymptomatic patients (including 17 patients with polyps detected by screening flexible sigmoidoscopy) and reported sensitivities for detection of $\geqslant 1 \mathrm{~cm}$ polyps of $29 \%$ on two dimensional and $50 \%$ on three dimensional colography, ${ }^{12}$ on a per polyp basis. Spinzi et al, using virtual colonoscopy software with $5 \mathrm{~mm}$ collimation and a pitch of 2 , also reported a sensitivity of only $50 \%$ for detecting $\geqslant 1 \mathrm{~cm}$ diameter polyps. ${ }^{13}$

Retrospective analysis suggested that $6 / 7$ $\geqslant 1 \mathrm{~cm}$ polyps that were missed on CT pneumocolon were not visualised because of the adjacent rectal tube, excessive faecal fluid, or collapse of the affected colonic segment. Therefore, use of a thinner rectal catheter, and greater attention to emptying and distending all colonic segments might have improved our detection rate for $\geqslant 1 \mathrm{~cm}$ diameter polyps. This may also involve rescanning of collapsed segments of colon after further distension and patient repositioning. The descending colon is often collapsed on supine images alone and full prone and supine scanning is advisable, although this may increase the radiation dose. We did not find significant differences in the probability of polyp detection by polyp site within the colon. Three dimensional reconstruction may also be helpful for evaluation of uncertain lesions seen on two dimensional axial scans. ${ }^{6} 1112$

Extracolonic lesions have rarely been reported in other CT colon studies. Previous CT colography studies have used low milliamps, with supine and prone scanning of the whole abdomen and pelvis. Low milliamps allows additional prone scanning while maintaining an acceptable radiation dose but results in poor views of extracolonic lesions. Using standard milliamps for abdominal and pelvic CT and intravenous contrast, we detected extracolonic lesions which were relevant to management in $7 \%$ of patients.

The acceptability of CT pneumocolon compared with conventional colonoscopy has not previously been addressed. The main reason for the lower pain score reported after conventional colonoscopy compared with CT pneumocolon was probably intravenous sedation and analgesia administered during conventional colonoscopy but not CT pneumocolon. In addition, the large volume of carbon dioxide 
administered by rectal insufflation may have contributed to the discomfort experienced with CT pneumocolon. Despite this however, there was no significant difference in patient preference for each technique on questioning during the month after study.

Cheaper, quicker, and more efficient virtual colonoscopy software is being developed. The speed of CT scanning is increasing, and multislice scanners are available. Thus it may be feasible to perform spiral CT pneumocolon (with intravenous contrast, $5-8 \mathrm{~mm}$ collimation, and 3-4 $\mathrm{mm}$ reconstruction) where assessment of both the colon and abdomen are required, and to undertake selective virtual colonoscopy (low milliamps without intravenous contrast) where assessment of the colon only is required.

The poor sensitivity of spiral CT pneumocolon in polyp detection could be improved by undertaking flexible sigmoidoscopy in addition to CT pneumocolon. As $69 \%$ of polyps identified in this study were within reach $(60 \mathrm{~cm}$ from the anal verge) of the flexible sigmoidoscope, addition of flexible sigmoidoscopy to CT pneumocolon would have resulted in a sensitivity of $88 \%$ and specificity of $92 \%$ in identifying patients with colorectal neoplasms.

In conclusion, CT pneumocolon had high sensitivity and specificity for detecting invasive colorectal carcinomas but not colorectal polyps. In addition, CT pneumocolon provided clinically relevant information on carcinoma staging and extracolonic abnormalities that was not available from conventional colonoscopy. Improvements to CT colon technique, and combining CT pneumocolon with flexible sigmoidoscopy may increase the sensitivity for identifying patients with polyps.

Currently, the authors do not feel that there is a role for CT pneumocolon in the routine diagnosis of suspected colorectal neoplasms. However, a role can be envisaged in certain problem solving situations such as: in the elderly patient who is unable to tolerate sedation and with poor mobility, making barium enema technically difficult to perform; immediately following a failed colonoscopy where the colon is already distended and where barium enemas can also be technically difficult to perform, as the distended bowel prevents adequate barium coating of the mucosal wall; and thirdly, in those patients suspected of nonspecific intra-abdominal malignancies.

We thank the endoscopy doctors who performed the conventional colonoscopies, the endoscopy nurses (led by E Nunn), the radiographers (led by J Cousins and K Green) and the reception staff (J O'Gorman and C Lockwood) for their help in organising staff (J O'Gorman and C Lockwood) for their help in organising
this study. We are also indebted to C Glover, MA, CStat, for this study. We are
statistical advice.

1 Rex DK, Rahmani EY, Haseman JH, et al. Relative sensitivity of colonoscopy and barium enema for detection of colorectal cancer in clinical practice. Gastroenterology 1997;112:17-23.

2 McCarty RL. Colorectal cancer: the case for barium enema. Mayo Clin Proc 1992;67:253-7.

3 Waye JD. What is a gold standard for colon polyps? Gastroenterology 1997;112:292-306.

4 Damore LJ, Rantis PC, Vernava AM, et al. Colonoscopic perforations: etiology, diagnosis, and management. Dis Colon Rectum 1996;39:1308-14.

5 Amin Z, Boulos PB, Lees WR. Technical Report: Spiral CT pneumocolon for suspected colonic neoplasms. Clin Radiol pneumocolon for

6 Hara AK, Johnson CD, Reed JE, et al. Detection of colorectal polyps with CT colography: initial assessment of sensitivity and specificity. Radiology 1997;205:59-65.

7 Hara AK, Johnson CD, Reed JE, et al. Colorectal polyp detection with CT colography: two- versus threedimensional techniques (work in progress). Radiology 1996;200:49-54

8 Rex DK. CT and MR colography (virtual colonoscopy)status report. F Clin Gastroenterol 1998;27:199-203.

9 Glick S, Wagner JL, Johnson CD. Cost effectiveness of double contrast barium enema in screening for colorectal cancer. AfR Am f Roentgenol 1998;170:629-36.

10 Dachman AH, Lieberman J, Osnis RB, et al. Small simulated polyps in pig colon: sensitivity of CT virtual simulated polyps in pig colon: sensitivity

11 Fenlon HM, Nunes DP, Schroy PC, et al. A comparision of virtual and conventional colonoscopy for the detection of colorectal polyps. N Engl f Med 1999;341:1496-503.

12 Rex DK, Vining D, Kopecky KK. An initial experience with screening for colon polyps using spiral CT with or without CT colography (virtual colonoscopy). Gastrointest Endosc 1999;50:309-13.

13 Spinzi GC, Belloni GM, Meucci G, et al. Virtual colonoscopy versus colonoscopy: a randomised prospective blinded study. Gut 1999;3(suppl 1): E1 (abstract 01.02E). 